\title{
NOTA
}

\section{UTILIZAÇÃO DE CERA DE ABELHAS NA DETERMINAÇÃO DA DENSIDADE DO SOLO ${ }^{(1)}$}

\author{
E. M. B. SILVA ${ }^{(2)}$, T. J . A. SILVA(2), L. B. OLIVEIRA (3), \\ R. F. MÉLO(3) \& P. K.T. J ACOMINE(4)
}

\begin{abstract}
RESUMO
Muitos têm-se empenhado em desenvolver métodos e técnicas de análises de solos que forneçam resultados confiáveis e a baixos custos, envolvendo, para isto, a reciclagem de materiais diversos ou o aproveitamento de produtos naturais que, ao contrário dos sintéticos, tendem a ter um baixo custo ambiental. A cera de abelhas atende, pelo menos, ao último destes requisitos. Neste trabalho, pretendeu-se realizar a caracterização física da cera de abelhas e avaliar a possibilidade de sua uti lização como revestimento impermeável, em substituição total a parafina, na determinação da densidade do solo. Para determinar a densidade do solo, utilizou-se o método do torrão impermeabilizado. 0 delineamento experimental utilizado foi inteiramente casualizado em arranjo fatorial $6 \times 2 \times 2$, sendo seis tipos de solos, dois revestimentos e dois diâmetros médios de torrões, com três repetições. A cera de abelhas apresentou características físicas semelhantes às da parafina, principalmente no que se refere a ponto de fusão, tempo de soli di ficação e densi dade específica. Os valores obtidos para densidade do solo, utilizando cera de abel has como revestimento impermeável, não diferiram estatisticamente dos valores obtidos utilizando parafina como revestimento. Desta forma, a parafina pode ser substituída total mente pela cera de abelhas na i mpermeabilização de torrões para determinar a densidade do solo, sem prejuízos aos resultados.
\end{abstract}

Termos de indexação: método de análise, Api s mellifera, características físicas, impermeabilização, parafina.

(1) Trabalho apresentado no XXVIII Congresso Brasileiro de Ciência do Solo. Recebido para publicação em setembro de 2001 e aprovado em maio de 2003.

(2) Doutorando do Programa de Pós-Graduação em Sol os e Nutrição de Plantas da Escola Superior de Agricultura "Luiz de Queiroz" - ESALQ. Av. André Vidal de Negreiros 30-A, Centro, CEP 55900-000 Goiana (PE). E-mail: agro_solo@yahoo.com.br

(3) Engenheiro(a)-Agrônomo(a) da Universidade Federal Rural de Pernambuco - UFRPE. Av. Dom Manuel de Medeiros s/n, Dois Irmãos, CEP 52171-900 E-mail: Ibdeoliveira@yahoo.com.br; fmralini@yahoo.com.br

(4) Professor Visitante do Departamento de Agronomia da, UFRPE. E-mail: ricardoj@interway.com.br 


\title{
SUMMARY: UTILIZATION OF BEESWAXIN PROCEDURES OF SOIL BULK DENSITY DETERMINATION
}

\begin{abstract}
Analysis methods and techniques in largenumbers which bring forth trustworthy results at low costs have been developed, some of which imply therecycling of diversified materials or the use of natural products. These, opposite to synthetic ones, normally have a low environmental cost. Beeswax fulfills, at least, this last condition. Our study had theaim of characterizing the physical properties of beeswax, and evaluating the possibility of using it as an impermeablecovering in thedetermination of soil bulk density, substituting theparaffin wax used in the clod method. The experimental design was completely randomized in a factorial arrangement of $6 \times 2 \times 2$ (six soils, two coverings, and two clod sizes), in three replicates. The physical characteristics of beeswax weresimilar to paraffin wax, especially regarding to the fusion point, solidification time and specific density. The values of soil bulk density with either beeswax or paraffin did not differ significantly. Thus, beeswax can substituteparaffin wax compl etely to seal soil cl ods in thedetermination of soil bulk density, without altering the results.
\end{abstract}

Index terms: analysis method, Apis mel lifera, physical characteristics, impermeablecovering, paraffin.

\section{INTRODUÇÃO}

A densidade do solo é um importante atributo físico dos sol os, por fornecer indicações a respeito do estado de sua conservação, sendo uma das primeiras propriedades a ser alterada pelos diferentes usos. Tem sido largamente utilizada na avaliação da compactação e, ou, adensamento dos sol os (Camargo, 1983; Dias J unior, 2000), sendo seu conhecimento necessário para conversão de resultados obtidos em base gravimétrica para volumétrica.

No Brasil, os três principais métodos adotados para determinação da densidade são: o do cilindro (ou anel) vol umétrico, o do torrão impermeabilizado e o da proveta (EMBRAPA, 1997). Trabal hos como os de Araújo Filho (1992) e de Silva (2000) mostram que os dois primei ros produzem resultados similares; assim, a escolha de um ou outro fica basicamente limitada pel o tipo de sol o queestiver sendo estudado. Sol os de el evada densi dade ou cascal hentos oferecem dificuldades à penetração do cilindro, podendo gerar compactação adicional nas paredes do cilindro ou neleproduzir deformações. Por outrolado, sol os que apresentam estrutura em grãos simples não fornecem agregados manuseáveis à determinação da densidade pel o método do tor rão impermeabilizado. O método da proveta só é recomendado para solos de textura arenosa, pois, por usar amostra deformada, não considera a estrutura original dos solos.

Quandoseutiliza ométodo do torrão impermeabilizado, atenção especial deve ser dada à escolha da substância usada como revestimento, que não deve permitir o umedecimento do torrão durante o processo de determinação do volume do solo e nem ser absorvida por ele. Tradicionalmente, a resina
Saran ou a parafina, ambas produtos sintéticos derivados do petróleo, têm sido utilizadas como revestimento de torrões de solo (Tan, 1996; EMBRAPA, 1997).

Dentre as substâncias naturais, disponíveis no mercado, que podem ser utilizadas em substituição a estes derivados do petróleo, destaca-se a cera de abelhas. Esta é uma substância secretada pelas abelhas através de quatro pares de glândulas serígenas, local izadas na parte inferior do abdômen (Kerr \& Amaral, 1960) e, para a apicultura, é considerada um subproduto. A produção de cera por uma colméia é variável; no entanto, estima-se que, em boas condi ções de produtividade, fique em torno de $10,8 \mathrm{~kg} \mathrm{ano}^{-1}$, tomando como referência uma proporção de $1 \mathrm{~kg}$ de cera para cada $10 \mathrm{~kg}$ de mel (Kerr \& Amaral, 1960; Wiese, 1995).

Considerando por hipótese a existência de semelhanças físicas entre a cera de abel has e a parafina e que aquela pode substituí-la na determinação da densidade de solos, realizou-se o presente trabalho com os seguintes objetivos: (a) caracterizar fisicamente a cera de abel has em comparação com a parafina; (b) testar sua utilização como revestimento impermeável de torrões de solo para determinação de sua densi dade.

\section{MATERIAL E MÉTODOS}

Para a caracterização física da cera de abel has e da parafina, foram avaliados o ponto e tempo de fusão, o tempo de solidificação e a densidade específica. Na obtenção do ponto e tempo de fusão, bequeres com cera ou parafina foram colocados em estufa a $105^{\circ} \mathrm{C}$, sendo observadas as mudanças de 
estado e cronometrado o tempo. O tempo de solidificaçãofoi contado desdeomomento da retirada dos bequeres da estufa, até não mais ser possível penetrar a massa com um bastão de vidro, à temperatura ambiente $( \pm 28 \stackrel{\circ}{\circ})$. Para determinar a densidade específica, foram utilizados "tabletes" de cera ou parafina, com tamanho médio de $5 \times 5 \times 3 \mathrm{~cm}$, empregando-se o mesmo método usado para determinação da densidade dos solos.

Para avaliar a espessura da camada de revestimento, utilizou-se a relação volume total do torrão impermeabilizado/volume do revestimento. O volume do revestimento foi obtido de acordo com a massa e com a densidade específica do revestimento (cera ou parafina) utilizado na impermeabilização do torrão, conforme EMBRAPA (1997). Espera-se que quanto mais delgada for a espessura da camada de revestimento, mais precisos sejam os resultados e mais econômica se torne a determinação.

Os sol os utilizados foram sel ecionados da col eção de Solos de Referência do Estado de Pernambuco. Os perfis foram descritos de acordo Lemos \& Santos (1996), sendo as amostras coletadas por horizonte, e classificados conforme EMBRAPA (1999). Algumas de suas características físicas e capacidade de troca de cátions são apresentadas no quadro 1. $\mathrm{Na}$ determinação destas características, foram adotados os métodos recomendados pela E MBRAPA (1997), tendo sido a densidade do solo obtida pelo método do torrão parafinado; a densidade de partículas, pelo método do balão volumétrico; a granulometria, pelo método do densímetro, e a capacidade de troca de cátions (CTC), pela soma das bases trocáveis mais hidrogêni o ealumínio trocáveis. A porosidade total foi calculada pela relação entre a densidade do solo e a densidade de partículas, conforme EMBRAPA (1997). A densidade dos solos selecionados estava compreendida entre 0,58 e $1,98 \mathrm{t} \mathrm{m}^{-3}$, valores estes que abrangem uma faixa representativa de densidade de solos.

O delineamento experimental utilizado foi inteiramente casualizado em arranjo fatorial
$6 \times 2 \times 2$, sendo seis sol os, dois diâmetros médios de torrões (3 e $5 \mathrm{~cm}$ ) e dois revestimentos, conforme Silva \& Silva (1999), com três repetições. Foi adotado o teste deTukey a $5 \%$, para comparação das médias, utilizando-se osoftwareSANEST (Zonta \& Machado, 1980).

\section{RESULTADOS E DISCUSSÃO}

A cera de abel has apresentou características físicas semelhantes às da parafina (Quadro 2). Resultados semelhantes para ponto de fusão, densidade específica ecor da cera foram registrados por Kerr \& Amaral (1960) e Wiese (1995). A similaridade das propriedades físicas dos dois produtos foi, também, observada na qualidade do revestimento. A cera, assim como a parafina, produziu um revestimento uniforme, pouco espesso eimpermeável à água, queénecessário para viabilizar a acurácia da determinação.

Os valores determinados para densidade do solo, de modo geral, não diferiram, estatisticamentea $5 \%$ pelo teste de Tukey, quando se utilizou a cera de abel has (ou a parafina) como revestimento impermeável (Quadro 3).

A reprodutibilidade dos resultados, quando da utilização da cera de abel has, valida a sua utilização como revestimento impermeável de torrões de solo em substituição à parafina na determinação da densidade do solo.

Deforma geral, não houve influência do diâmetro médio do torrão na determinação da densidade dos sol os, independentemente do revestimento utilizado na determinação (Quadro 3). De acordo com EMBRAPA (1997), torrões com diâmetros médios entre 4 e $7 \mathrm{~cm}$ são mais indicados para a determinação da densidade de solos. Torrões menores do que $5 \mathrm{~cm}$ apresentam dificuldades no manuseio, estão mais sujeitos a erros operacionais e podem ser pouco representativos, sobretudo em

Quadro 1. Alguns atributos físicos e capacidade de troca de cátions dos horizontes dos solos utilizados neste estudo

\begin{tabular}{|c|c|c|c|c|c|c|c|c|c|c|}
\hline Classe & Localidade & Horizonte & Profundidade & Areia & Silte & Argila & Ds ${ }^{(1)}$ & $\mathbf{D p}^{(2)}$ & $\mathbf{P t}^{(3)}$ & СTC \\
\hline & & & $\mathrm{cm}$ & - & $\mathrm{g} \mathrm{kg}^{-1}$ & - & \multicolumn{2}{|c|}{$-\mathrm{t} \mathrm{m}^{-3}-$} & $\%$ & $\mathrm{cmol}_{\mathrm{c}} \mathrm{kg}^{-1}$ \\
\hline Organossolo & I pojuca & Ap & $0-20$ & - & - & - & 0,58 & - & - & 33,3 \\
\hline Argissolo Vermelho & Camutanga & $\mathrm{Bt}_{1}$ & 56-105 & 168 & 200 & 632 & 1,52 & 2,67 & 43 & 4,3 \\
\hline Gleissolo & I pojuca & $\mathrm{Cg}_{1}$ & $42-70$ & - & - & - & 1,63 & - & - & 25,7 \\
\hline Luvissolo Crômico & Cabrobó & $2 \mathrm{Bt}$ & $23-46$ & 400 & 139 & 461 & 1,44 & 2,64 & 45 & 19,5 \\
\hline Vertissolo Cromado & Bodocó & Biv & $25-65$ & 248 & 127 & 625 & 1,57 & 2,70 & 42 & 41,9 \\
\hline Planossolo Nátrico & Petrolina & $\mathrm{Btn}_{2}$ & $55-80$ & 550 & 113 & 337 & 1,98 & 2,61 & 25 & 10,9 \\
\hline
\end{tabular}

${ }^{(1)}$ Densidade do solo. ${ }^{(2)}$ Densidade de partículas. ${ }^{(3)}$ Porosidade total calculada. 
Quadro 2. Características físicas da cera de abelhas e da parafina

\begin{tabular}{lccc}
\hline \multicolumn{1}{c}{ Característica física } & Cera de abelha & Parafina & C.v. \\
& & & $\%$ \\
Ponto de fusão $\left({ }^{\circ} \mathrm{C}\right)$ & $62,5 \mathrm{a}(1)$ & $62,5 \mathrm{a}$ & 2,70 \\
Tempo de fusãa $(\mathrm{min})$ à $105 \stackrel{\circ}{\circ} \mathrm{C}$ & $110 \mathrm{a}$ & $100 \mathrm{a}$ & 7,20 \\
Tempo de solidificação $(\mathrm{min})$ & $40 \mathrm{a}$ & $40 \mathrm{a}$ & 2,51 \\
Densidade específica $\left(\mathrm{t} \mathrm{m}^{-3}\right)$ & $0,95 \mathrm{a}$ & $0,90 \mathrm{a}$ & 2,58 \\
Cor & branca ou amarela & branca &
\end{tabular}

(1) Médias seguidas por letras iguais, na mesma linha, não diferem estatisticamente entre si, a 5 \% pelo teste de Tukey.

Quadro 3. Densidade dos solos obtida com diferentes tamanhos de torrões e tipos de revestimentos

\begin{tabular}{|c|c|c|c|c|}
\hline \multirow{2}{*}{ Classe de solo } & \multirow{2}{*}{ Diâmetro médio do torrão } & \multicolumn{2}{|c|}{ Revestimento } & \multirow{2}{*}{ C.v. } \\
\hline & & Cera & Parafina & \\
\hline & $\mathrm{cm}$ & 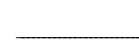 & - & $\%$ \\
\hline Organossolo & $\begin{array}{l}3 \\
5\end{array}$ & $\begin{array}{l}0,64 \mathrm{Aa} \\
0,76 \mathrm{Aa}\end{array}$ & $\begin{array}{l}0,71 \mathrm{Aa} \\
0,64 \mathrm{Aa}\end{array}$ & $\begin{array}{r}13,57 \\
6,31\end{array}$ \\
\hline Argissolo Vermelho & $\begin{array}{l}3 \\
5\end{array}$ & $\begin{array}{l}1,63 \mathrm{Aa} \\
1,60 \mathrm{Aa}\end{array}$ & $\begin{array}{l}1,60 \mathrm{Aa} \\
1,55 \mathrm{Aa}\end{array}$ & $\begin{array}{l}5,72 \\
2,93\end{array}$ \\
\hline Gleissolo & $\begin{array}{l}3 \\
5\end{array}$ & $\begin{array}{l}1,66 \mathrm{Aa} \\
1,66 \mathrm{Aa}\end{array}$ & $\begin{array}{l}1,66 \mathrm{Aa} \\
1,61 \mathrm{Ab}\end{array}$ & $\begin{array}{l}3,35 \\
1,76\end{array}$ \\
\hline Luvissolo Crômico & $\begin{array}{l}3 \\
5\end{array}$ & $\begin{array}{l}1,71 \mathrm{Aa} \\
1,76 \mathrm{Aa}\end{array}$ & $\begin{array}{l}1,67 \mathrm{Aa} \\
1,68 \mathrm{Ba}\end{array}$ & $\begin{array}{l}2,94 \\
3,04\end{array}$ \\
\hline Vertissolo Cromado & $\begin{array}{l}3 \\
5\end{array}$ & $\begin{array}{l}1,70 \mathrm{Aa} \\
1,79 \mathrm{Ab}\end{array}$ & $\begin{array}{l}1,70 \mathrm{Aa} \\
1,77 \mathrm{Aa}\end{array}$ & $\begin{array}{l}2,39 \\
1,82\end{array}$ \\
\hline Planossolo Nátrico & $\begin{array}{l}3 \\
5\end{array}$ & $\begin{array}{l}1,88 \mathrm{Aa} \\
2,02 \mathrm{Aa}\end{array}$ & $\begin{array}{l}1,91 \mathrm{Aa} \\
2,05 \mathrm{Aa}\end{array}$ & $\begin{array}{l}2,74 \\
1,44\end{array}$ \\
\hline
\end{tabular}

(1) Médias seguidas por mesmas letras maiúsculas na mesma linha, e por mesma letra minúscula na mesma coluna não diferem estatisticamente entre si, a $5 \%$ pelo teste de Tuckey.

sol os com estrutura muito pequena. Por outrolado, os maiores do que $8 \mathrm{~cm}$, além das dificuldade de manuseio, principalmente em solos cujo grau de desenvol vimento da estrutura éfraco, consomem um volume mai or de impermeabilizante, gerando custos adicionais na determinação, que não são, necessariamente, justificados por maior exati dão dos resultados.

Com relação à espessura do revestimento, analisada por meio da relação vol ume total do torrão impermeabilizado/volume do revestimento, de modo geral, a cera produziu revestimentos mais espessos do quea parafina. Não foram constatadas diferenças para espessura do revestimento quando da utilização de torrões com diâmetro médio de $5 \mathrm{~cm}$ para o
Argissolo Vermel ho, Luvissolo Crômicoe Vertissolo Cromado. O mesmo aconteceu para o Planossolo Nátrico, quando se utilizaram torrões de diâmetro médio de $3 \mathrm{~cm}$ (Quadro 4). O uso de parafina ou cera próximo ao ponto de solidificação, no momento da imersão dotorrão, diminui as chances de absorção desses produtos pelo solo.

Quando a resina Saran é utilizada como revestimento em solos com predomínio de macroporos, a proporção da mistura resina: solvente deve ser diminuída de 1:8 para 1:4 (Tan, 1996). Quando da utilização da resina Saran, a escol ha do solvente é, também, importante, daí o metil-etilcetona ser preferido, em relação à acetona, uma vez que é menos solúvel em água (Tan, 1996). 


\section{Quadro 4. Relação volume total do torrão impermeabilizado/volume do revestimento para os diferentes solos}

\begin{tabular}{|c|c|c|c|c|}
\hline \multirow{2}{*}{ Classe de solo } & \multirow{2}{*}{ Diâmetro médio do torrão } & \multicolumn{2}{|c|}{ R evestimento } & \multirow{2}{*}{ C.V. } \\
\hline & & Cera & Parafina & \\
\hline & $\mathrm{cm}$ & & & $\%$ \\
\hline Organossolo & $\begin{array}{l}3 \\
5\end{array}$ & $\begin{array}{l}5,61 a \\
5,60 a\end{array}$ & $\begin{array}{l}8,26 \mathrm{~b} \\
9,01 \mathrm{~b}\end{array}$ & $\begin{array}{l}27,74 \\
26,63\end{array}$ \\
\hline Argissolo Vermel ho & $\begin{array}{l}3 \\
5\end{array}$ & $\begin{array}{l}7,54 a \\
6,08 a\end{array}$ & $\begin{array}{r}10,82 \mathrm{~b} \\
6,35 \mathrm{a}\end{array}$ & $\begin{array}{l}20,83 \\
11,74\end{array}$ \\
\hline Gleissolo & $\begin{array}{l}3 \\
5\end{array}$ & $\begin{array}{r}8,59 \text { a } \\
11,15 \text { a }\end{array}$ & $\begin{array}{l}11,98 \mathrm{~b} \\
15,80 \mathrm{~b}\end{array}$ & $\begin{array}{l}20,88 \\
27,51\end{array}$ \\
\hline Luvissolo Crômico & $\begin{array}{l}3 \\
5\end{array}$ & $\begin{array}{l}5,44 \mathrm{a} \\
4,74 \mathrm{a}\end{array}$ & $\begin{array}{l}7,34 \mathrm{~b} \\
5,24 \mathrm{a}\end{array}$ & $\begin{array}{l}18,69 \\
27,51\end{array}$ \\
\hline Vertissolo Cromado & $\begin{array}{l}3 \\
5\end{array}$ & $\begin{array}{l}6,35 a \\
5,11 a\end{array}$ & $\begin{array}{l}8,37 \mathrm{~b} \\
6,65 \mathrm{a}\end{array}$ & $\begin{array}{l}19,93 \\
15,81\end{array}$ \\
\hline Planossolo Nátrico & $\begin{array}{l}3 \\
5\end{array}$ & $\begin{array}{l}9,03 a \\
6,26 a\end{array}$ & $\begin{array}{l}8,33 \mathrm{a} \\
9,11 \mathrm{~b}\end{array}$ & $\begin{array}{r}7,07 \\
21,74\end{array}$ \\
\hline
\end{tabular}

(1) Médias seguidas por letras iguais, na mesma linha, não diferem estatisticamente entre si, a 5 \% pelo teste de Tuckey.

\section{CONCLUSÕES}

1. As características físicas analisadas (ponto de fusão, tempo de fusão e solidificação e densidade específica) da cera de abelhas foram semelhantes às da parafina.

2. A cera de abelhas pode ser utilizada como revestimento impermeável, em substituição à parafina, na determinação da densi dade desol os pel o método do torrão impermeabilizado.

\section{AGRADE CIMENTOS}

Os autores expressam seus agradecimentos a Anacleto J osé da $\mathrm{F}$ onseca J únior, técnico do laboratório de física do solo da UFRPE, pela ajuda prestada.

\section{LITE RATURA CITADA}

ARAÚJ O FILHO, J.C. Infiltração e disponibilidade hídrica em Cambissolos do Baixio de Irecê - Bahia. Recife, Universidade Federal Rural de Pernambuco, 1992. 224p. (Tese de Mestrado)

CAMARGO, A.O. Compactação do solo e desenvolvimento de plantas. Campinas, Fundação Cargill, 1983. 44p.
EMPRESA BRASILEIRA DE PESQUISA AGROPECUÁRIA EMBRAPA. Manual de métodos de análises de solo. 2.ed. Rio de Janeiro, Ministério da Agricultura e do Abastecimento, 1997. 212p.

EMPRESA BRASILEIRA DE PESQUISA AGROPECUÁRIA EMBRAPA. Serviço de Produção de I nformações. Sistema Brasileiro de Classificação de Solos. Brasília, 1999. 412p.

DIAS J ÚNIOR, M.S. Compactação do solo. In: NOVAIS, R.F.; ALVAREZ V., V.H. \& SHAEFER, C.E.G.R. Tópicos em ciência do solo. Viçosa, Sociedade Brasileira de Ciência do Solo, 2000. v.1. p.55-94.

KERR, E.W. \& AMARAL, E. Apicultura científica e prática. São Paulo, Indústrias Gráficas Siqueira, 1960. 148p.

LEMOS, R.C. \& SANTOS, R.D. Manual de descrição e col eta de solos no campo. 3.ed. Campinas, Sociedade Brasileira de Ciência do Solo, 1996. 84p.

SILVA, I.P. \& SILVA, J .A.A. Métodos estatísticos aplicados à pesquisa científica: uma abordagem para profissionais da pesquisa agropecuária. Recife, Universidade F ederal Rural de Pernambuco, 1999. 305p.

SILVA, M.S.L. Caracterização e gênese do adensamento subsuperficial em solos de tabuleiros do semi-árido do Nordeste do Brasil. Porto Alegre, Universidade F ederal do Rio Grande do Sul, 2000. 126p. (Tese de Doutorado)

WIESE, H. Novo manual de apicultura. Guaíba, Agropecuária, 1995. 295p.

TAN, K.H. Soil sampling, preparation and analysis. New York, Marcel Dekker, 1996. 408p.

ZONTA, E.P. \& MACHADO, A.A. SANEST - Sistema deAnálise Estatística. São Paulo, Escola Superior de Agricultura Luiz de Queiroz. 1980. Programa em disquete. 
E.M.B. SILVA et al. 